\title{
LES AILES DE GLOSSINES, UNE CARTE D'IDENTITÉ DE L'INSECTE ?
}

\author{
DE LA ROCQUE S.*,**, GEOFFROY B.***, MICHEL J.F.*,**, BORNE F.****, SOLANO P.***, \\ MEUNIER J.Y.*** \& CUISANCE D.*
}

Summary: TSETSE FLIES WINGS, AN IDENTITY CARD OF THE INSECT?

The size of tsetse flies is often associated with population dynamics and vectorial capacity parameters. Adult fly size is generally estimated from measurements of wing segments. To take measure of the wing, a semi-automatic software was developed by CIRAD-EMVT and IRD. It was used in wild populations of Glossina tachinoides Westwood and G. palpalis gambiensis Vanderplank (Diptera: Glossinidae) trapped near Bobo-Dioulasso, Burkina Faso. From an numeric picture of the wing, the software calculates the length of vein segments, the ratios between these lengths, the surface of the tsetse characteristic "hatchet cell", and the greyness on the wings. The data were interesting at the level of taxonomy. In addition, they help specify physiological

characteristics of the studied populations.

KEY WORDS : Glossina, size, wing, morphometry.

\section{Résumé :}

Dans les études de dynamique de population des glossines, vecteurs des trypanosomoses en Afrique, la taille de l'insecte peut être mise en relation avec leur longévité et leur capacité vectorielle. La taille des individus est généralement appréciée par la mesure de nervures remarquables de l'aile. Un logiciel semiautomatique a été développé par le Cirad et l'IRD pour réaliser une série de mesures à partir de photos numérisées des ailes. Il a été utilisé sur des populations sauvages de Glossina tachinoides Westwood et $G$. palpalis gambiensis Vanderplank (Diptera: Glossinidael capturées autour de Bobo Dioulasso, au Burkina Faso. A partir d'une photo de l'aile, réalisée sous une loupe binoculaire et numérisée, le logiciel calcule la longueur des segments alaires, le rapport entre ces segments, la surface de la cellule "en hache" caractéristique des glossines et le niveau de gris de la membrane. Ces variables ont révélé leur intérêt taxonomique pour la diagnose entre ces espèces, mais également apportent des informations sur les caractéristiques physiologiques de la population étudiée.

MOTS CLÉS : Glossina, taille, aile, morphométrie.
$\mathrm{D}$ es études morphométriques sur les insectes peuvent être utilisées dans les domaines de la systématique, la phylogénie, la génétique, l'écologie ou pour l'étude des dynamiques de population (Daly, 1985). Chez les glossines (Diptera: Glossinidae), les mesures sont généralement réalisées sur les ailes, organes dont la taille est proportionnelle à celle du corps (Bursell, 1960 ; Buxton, 1955). Dans le genre Glossina, l'aile a six veines longitudinales : les trois premières sont parallèles au côté costal, et la qua-

\footnotetext{
* Centre de Coopération Internationale en Recherche Agronomique pour le Développement, Département Élevage et Médecine Vétérinaire (CIRAD-EMVT), Campus de Baillarguet, BP 5035, 34032 Montpellier cedex 5, France.

** Centre International de Recherche-Développement sur l'Élevage en zone Subhumide (CIRDES), BP 454, Bobo Dioulasso, Burkina Faso.

*** Institut de Recherche pour le Développement (IRD), Département Santé, avenue Agropolis, BP 5045, 34032 Montpellier, France.

**** Centre de Coopération Internationale en Recherche Agronomique pour le Développement, Département Amélioration des Méthodes pour l'Innovation Scientifique (CIRAD-AMIS), avenue Agropolis, 34398 Montpellier cedex 5, France.

Correspondance : Stéphane de La Rocque.

Tél. : (226) 973687 - Fax : (226) 972320.

E-mail: stephane.delarocque (1)cirad.fr
}

trième et la cinquième forment une cellule discale "en hache" caractéristique. La longueur du tranchant de cette cellule (soit la partie moyenne de la quatrième veine longitudinale d'aile) est souvent employée comme seule mesure de la taille.

Le présent travail décrit l'utilisation d'un logiciel semiautomatique permettant d'obtenir différents paramètres morphométriques à partir d'une image de l'aile. Des variations de ces mesures sont discutées selon l'espèce, le sexe, la saison de la capture et d'autres paramètres écologiques.

\section{MATÉRIEL ET MÉTHODES}

T es insectes ont été capturés dans la zone agropastorale de Sidéradougou, près de Bobo Diouasso (Burkina Faso, Afrique occidentale) où sont présentes deux espèces riveraines du groupe Palpalis : Glossina tachinoides (Westwood 1850) et G. palpalis gambiensis (Vanderplank 1949). Durant la saison sèche de 1996, des pièges biconiques (Challier \& Laveissière, 1973) ont été placés tous les 100 mètres le long de 120 kilomètres de la rivière principale (le Koba) et de son 
principal affluent (le Tolé). Au niveau de chaque piège, 70 paramètres écologiques ont été enregistrés afin de caractériser les facteurs principaux corrélés avec la présence de chacune des espèces (de La Rocque, 1997). Parmi les 3600 mouches tsé-tsé capturées, 619 paires d'ailes ont été préparées selon la méthode décrite ci-dessous.

\section{PRÉPARATION DES SPÉCIMENS}

Chaque paire d'ailes a été montée à sec et sans préparation entre lame et lamelle; l'origine de l'aile (gauche ou droite) a été enregistrée de sorte que l'éventuelle asymétrie puisse être prise en compte (Bruckner, 1976). Les ailes ont été photographiées numériquement par un appareil-photo Canon XE1 monté sur une loupe binoculaire Olympus SZH (grossissement 75).

\section{Le logiciel "Fly Picture Measurement (FPM)"}

Les mesures ont été réalisées grâce au logiciel "Fly Picture Measurement" mis au point par le Cirad et l'Ird (Borne et al., 1999). Sur l'image numérique visualisée à l'écran, l'utilisateur sélectionne avec un clic de la souris huit points remarquables situés au niveau des intersections de nervures. Les cinq premiers points sont situés aux angles de la cellule en hache (fig. 1). Le logiciel détermine alors la surface de la cellule, et la taille (en millimètres) de trois segments entre les points 6 et 8 (segment 1), 3 et 7 (segment 2) et 2 et 8 (segment 3). Afin d'apprécier la conformation de l'organe, il calcule également les rapports entre segment 1 et segment 2, segment 1 et segment 3, segment 2 et segment 3 . Enfin, deux petits disques de $3 \mathrm{~mm}^{2}$ sont créés, à l'intérieur desquels est mesuré le niveau de gris (sur une échelle de 256 niveaux). Les disques sont posi- tionnés l'un sur la membrane de l'aile, l'autre à l'extérieur de l'organe mais sur la lamelle, afin de tenir compte de la variabilité due au verre ou à la lumière. La valeur retenue est la différence entre les deux niveau de gris.

Les résultats de toutes les mesures sont sauvegardées en format texte ou tabulaire. Avec un peu de pratique, deux à trois ailes peuvent ainsi être examinées en une minute. La présentation de ce logiciel est disponible sur internet à l'adresse suivante :

http://www.multimania.com/bornef/FPM/FPM.htm

\section{TRAITEMENT DES DONNÉES}

L'analyse de variables très corrélées entre elles est redondante. Une manière de résumer les informations qu'elles apportent consiste à effectuer une analyse en composantes principales (ACP) et à décrire une variable de synthèse correspondant à une combinaison linéaire des variables d'origine (Mardia et al., 1979). Les variations morphométriques selon l'espèce, le sexe et le milieu fréquenté ont été examinés. Les distributions ont été comparées aux distributions normales (test de Kolmogorrov-Smirnov), puis la comparaison des moyennes a été réalisée par analyse de variance (ANOVA). Tous les analyses statistiques ont été réalisées avec le logiciel S-plus ${ }^{\mathrm{tm}}$.

\section{RÉSULTATS}

T es variables concernant la taille de l'organe (la surface de cellule en hache et les segments 1 à 3) 1 se sont révélées fortement corrélées $(r>0,86)$. L'ACP montre que 94,6\% de la variabilité de ces données est expliquée par le premier axe de l'analyse. Les

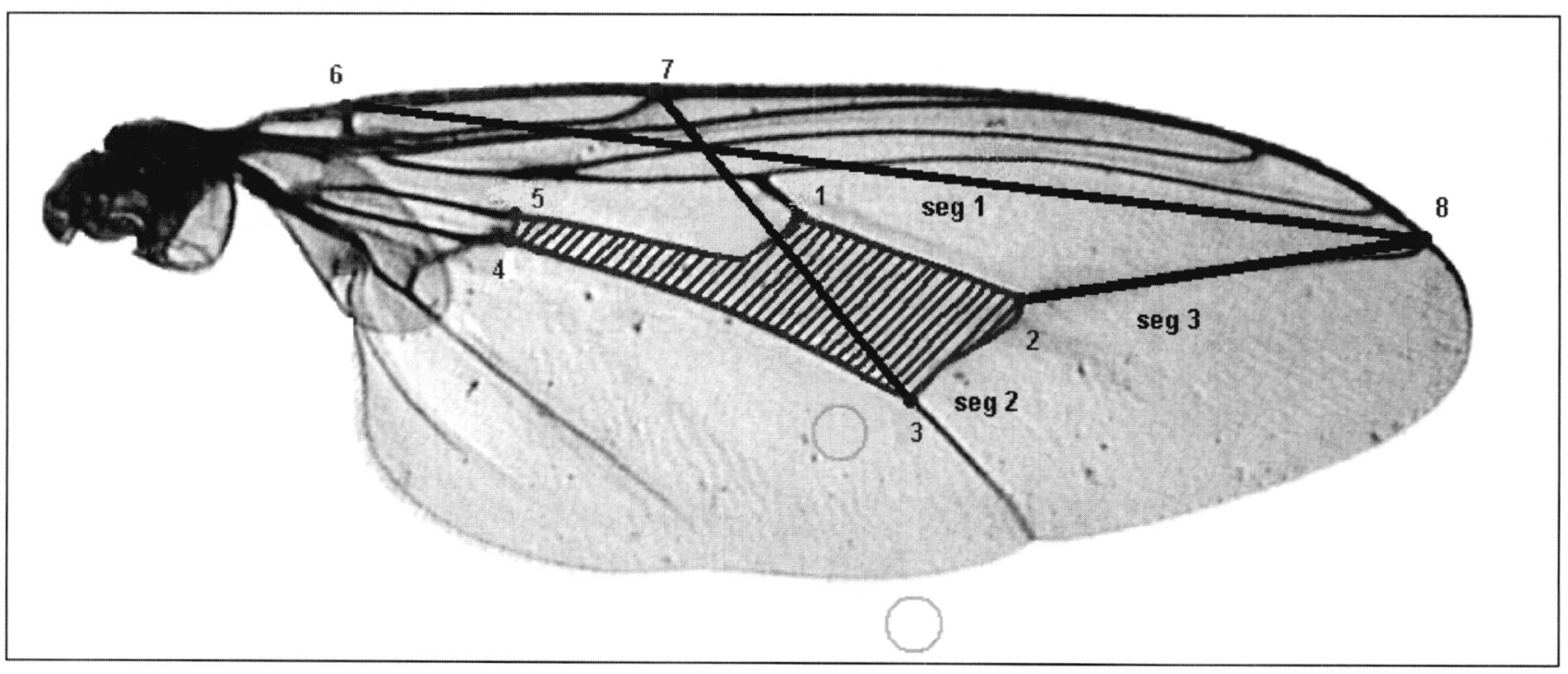

Fig. 1. - Mesures alaires : localisation des points à sélectionner et longueurs calculées. 
Fig. 2a. Variable synthétique
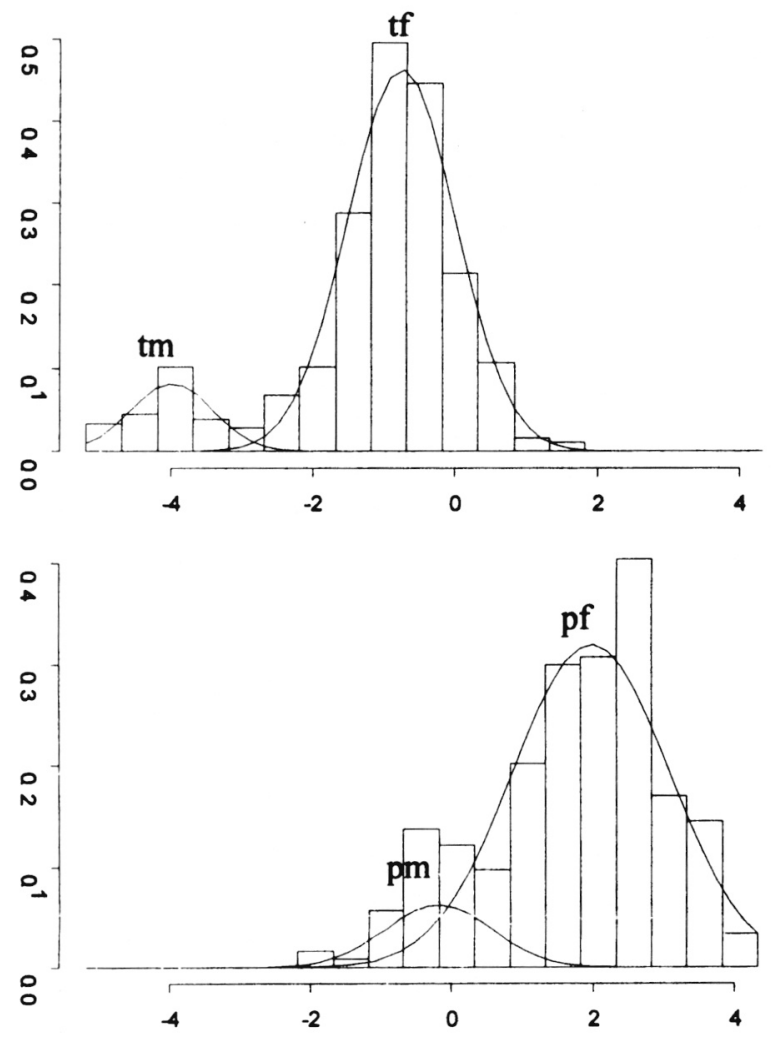

Fig. 2b. Niveau de gris
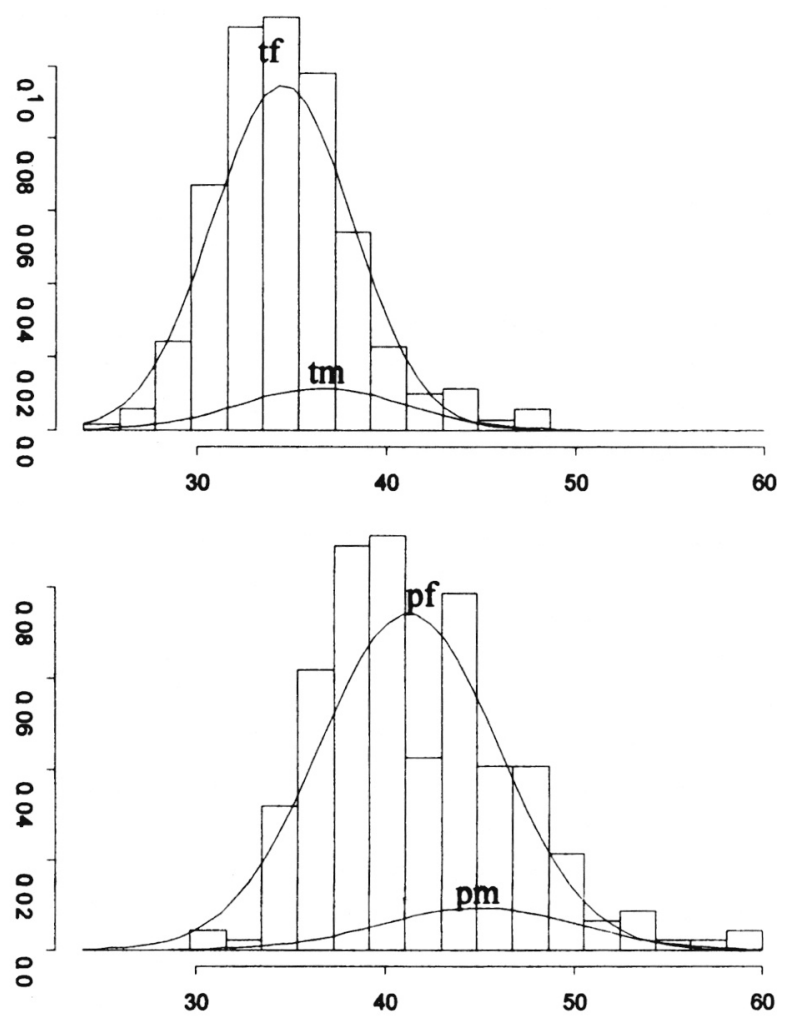

Fig. 2c. Rapport segment $2 /$ segment 3
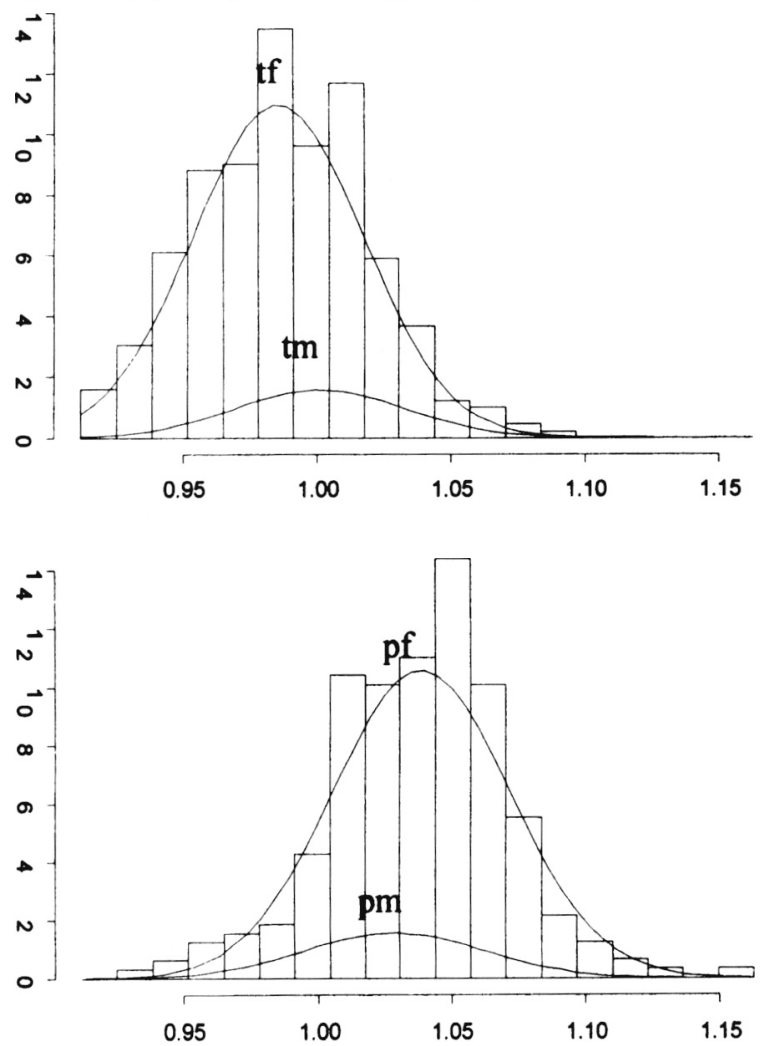

Fig. 2. - Distribution des paramètres morphométriques selon l'espèce et le sexe (en histogramme) et lois normales. $\mathrm{tf}=G$. tachinoides femelle, $\mathrm{tm}=G$. tachinoides mâle, $\mathrm{pf}=G$. pal palis gambiensis femelle, $\mathrm{pm}=G$. palpalis gambiensis mâle. 
coefficients de la combinaison linéaire, utilisés pour définir la variable synthétique, sont respectivement $0,50,0,51,0,50$ et 0,49 , ce qui reflète l'importance égale de ces quatre variables. Les autres variables (rapports entre les tailles des segments, niveau de gris) sont indépendantes.

\section{DifFÉRENCES TAXONOMIQUES}

L'influence du sexe et de l'espèce ont été testés de manière simultanée. Sur la figure 2 sont représentées les distributions de la variable et les distributions normales associées. Cette figure illustre plusieurs résultats importants i) la normalité des distributions, confirmées par le test de Kolmogorov ( $\mathrm{p}>0,05$ ), ii) l'homogénéité des variances, $i i i$ ) les différences entre espèces et sexe détaillées dans le tableau I. L'analyse de variance a confirmé que les ailes de G.p. gambiensis sont plus larges $(\mathrm{F}=1000)$, et que celles des mâles sont plus petites dans les deux espèces $(F=424)$ mais surtout chez G. tachinoides (fig. 2a).

Les rapports entre les segments varient également entre les deux espèces, indiquant des différences dans la taille et la conformation de l'organe. L'aile de G.p. gambiensis apparaît plus large et ovale (rapport $1 / 2$ plus faible $(\mathrm{F}=308)$ et rapport $2 / 3$ plus élevé $(\mathrm{F}=$ 348)), avec une partie distale plus courte (rapport $1 / 3$ plus élevé, $F=163$ ). En revanche, à l'intérieur de chaque espèce, ces rapports sont constants.

\section{VARIATIONS SAISONNIERES}

Cette analyse a été limitée aux femelles de G. tachinoides, pour lesquelles les effectifs étaient suffisants (tableau II). La prospection ayant été exhaustive tout le long du réseau hydrographique, il a en effet fallu se limiter aux captures réalisées dans des milieux écologiquement comparables pour limiter l'effet des autres paramètres environnementaux.

Les individus ont été prélevés à différentes dates dans des galeries forestières ayant les mêmes caractéristiques écologiques. Des variations significatives de la variable synthétique $(\mathrm{F}=4,2, \mathrm{p}<0,05)$ indiquent un effet saisonnier sur la taille d'aile, essentiellement lié à une diminution entre janvier et avril $(\mathrm{F}=5,9, \mathrm{p}<$ 0,05). Ces deux mois correspondent à deux périodes climatiques différentes, le premier situé durant la saison sèche fraîche, et le second au milieu de la saison sèche chaude. Les niveaux de gris et les rapports entre les segments n'ont pas varié.

\section{VARIATIONS SELON LE TYPE D'HABITAT}

Deux sites de captures, séparés d'une distance de 15 kilomètres ont été prospectés consécutivement (15-17 et 25-26 avril). Le premier site se localise sur la partie ouest du Koba et correspond à une forêt galerie bordant la rivière en eau dix mois par an. Le second se situe sur le Tolé, dans un cordon ripicole étroit à sec durant plus de la moitié de l'année. Les deux sites

\begin{tabular}{|c|c|c|c|c|}
\hline & \multicolumn{2}{|c|}{ G. p. gambiensis } & \multicolumn{2}{|c|}{ G. tachinoides } \\
\hline & Femelles & Mâles & Femelles & Mâles \\
\hline $\mathrm{n}$ & 218 & 30 & 324 & 47 \\
\hline Surface de la cellule en hache & $1,3 \quad(0,1)$ & $1,1 \quad(0,09)$ & $1,1 \quad(0,08)$ & $0,9 \quad(0,1)$ \\
\hline Niveau de gris & $41,2 \quad(4,7)$ & $44,9 \quad(5,2)$ & $34,5 \quad(3,7)$ & $36,6 \quad(4,4)$ \\
\hline Segment 1 & $6,2 \quad(0,2)$ & $5,8 \quad(0,2)$ & $5,7 \quad(0,2)$ & $5,1 \quad(0,25)$ \\
\hline Segment 2 & $2,3 \quad(0,09)$ & $2,1 \quad(0,1)$ & $2,1 \quad(0,08)$ & $1,9 \quad(0,1)$ \\
\hline Segment 3 & $2,2 \quad(0,08)$ & $2,1 \quad(0,09)$ & $2,1 \quad(0,08)$ & $1,9 \quad(0,09)$ \\
\hline Variable synthétique & $1,9 \quad(1,1)$ & $-0,2 \quad(0,8)$ & $-0,75(0,8)$ & $-5,1 \quad(0,6)$ \\
\hline Ratio $1 / 2$ & $2,66(0,06)$ & $2,70(0,05)$ & $2,75(0,06)$ & $2,72(0,05)$ \\
\hline Ratio $1 / 3$ & $2,76(0,05)$ & $2,78(0,04)$ & $2,70(0,05)$ & $2,72(0,05)$ \\
\hline Ratio $2 / 3$ & $1,04(0,03)$ & $1,03(0,03)$ & $0,99(0,03)$ & $1 \quad(0,03)$ \\
\hline
\end{tabular}

Tableau I. - Moyenne et écarts-type (entre parenthèses) selon l'espèce et le sexe des mesures réalisées sur les ailes ( $\mathrm{n}=$ nombre d'individus, surface en $\mathrm{mm}^{2}$, longueur des segments en $\mathrm{mm}$, niveau de gris entre 0 et 255 ).

\begin{tabular}{lllllll}
\hline & $\mathbf{n}$ & $\begin{array}{c}\text { Variable } \\
\text { synthétique }\end{array}$ & $\begin{array}{l}\text { Niveau } \\
\text { de gris }\end{array}$ & Ratio 1/2 & Ratio 1/3 & Ratio 2/3 \\
\hline Janvier (saison sèche froide) & 19 & $-0,2(1)$ & $34,9(4,2)$ & $2,72(0,05)$ & $2,72(0,05)$ & 1 \\
Avril (saison sèche chaude) & 24 & $-0,9(1)$ & $34,6(3,7)$ & $2,73(0,06)$ & $2,71(0,06)$ \\
Mai (saison sèche chaude) & 73 & $-0,7(0,6)$ & $34,8(3,8)$ & $2,76(0,06)$ & $2,71(0,05)$ & $0,99(0,03)$ \\
\hline
\end{tabular}

Tableau II. - Moyenne et écarts-type (entre parenthèses) des mesures morphométriques obtenues avec des individus de Glossina tachinoides femelles capturées à différentes dates dans la zone de Sidéradougou $\left(\mathrm{n}=\right.$ nombre d'individus, surface en $\mathrm{mm}^{2}$, niveau de gris entre 0 et 255). 


\begin{tabular}{lccccrr}
\hline & n & $\begin{array}{c}\text { Variable } \\
\text { synthétique }\end{array}$ & $\begin{array}{c}\text { Niveau } \\
\text { de gris }\end{array}$ & Ratio 1/2 & Ratio 1/3 & Ratio 2/3 \\
\hline Tole & 49 & $-1,1(0,7)$ & $34,2(10,6)$ & $2,75(0,003)$ & $2,69(0,002)$ & $0,98(0,001)$ \\
Koba & 32 & $-0,36(1)$ & $34,1(11,7)$ & $2,73(0,003)$ & $2,71(0,005)$ & $0,99(0,001)$ \\
\hline
\end{tabular}

Tableau III. - Moyenne et écarts-type (entre parenthèses) des mesures morphométriques obtenues avec des individus de Glossina tachinoides femelles capturées le long des rivières Tolé et Koba : $\left(\mathrm{n}=\right.$ nombre d'individus, surface en $\mathrm{mm}^{2}$, niveau de gris entre 0 et 255 ).

hébergent des populations de G. tachinoides de manière permanente (de La Rocque, 1997). Les résultats des mesures apparaissent dans le tableau III. L'effet géographique est nettement significatif sur la variable synthétique $(\mathrm{F}=13,6)$, l'aile des mouches capturées sur le Koba étant plus grande. En revanche le niveau gris et les rapports de segments sont comparables entre les deux emplacements.

\section{DISCUSSION}

\section{INTÉRET TAXONOMIQUE \\ DES MESURES MORPHOMÉTRIQUES SUR LES AILES}

$\mathrm{E}$ n entomologie, des mesures morphométriques sont réalisées sur différentes parties du corps, notamment les ailes. Par exemple, Weeks et al. (1997) ont pu discriminer cinq groupes très proches d'Ichneumonidae (hyménoptères parasites) à partir d'images d'ailes typiques construites en utilisant un logiciel de reconnaissance de formes. Les mesures de différents segments alaires permettent également des diagnoses au sein des Muscidae, des Sarcophagidae, des Calliphoridae (Brown, 1979) et des Phlebotomidae (Dujardin et al., 1997; Lebbe et al., 1991; Passerat de Silans, 1994). Comparées à celles d'autres familles de diptères, les variations de taille observées dans les populations de tsé-tsé sont assez limitées. Cette observation est à rapprocher de leur caractère pupipare, qui assure à la larve des conditions privilégiées de développement, notamment au niveau nutritionnel (Glasgow, 1961). Cette variabilité limitée est illustrée dans nos résultats par des écarts-type faibles lorsque les insectes sont regroupés par espèce et par le sexe.

Les résultats sur la morphologie des ailes se sont avérés efficaces pour différencier les deux espèces, la taille étant le paramètre le plus fiable. Dans la littérature, des différences interspécifiques ont également été décrites sur d'autres structures anatomiques, jusque sur la taille des follicules ovariens (Wall, 1990). La forme des ailes est un critère moins pertinent, mais est indépendante d'autres facteurs tels que la saison, le sexe et le milieu de vie. Au sein de chaque espèce, il est également possible de différencier le sexe de l'individu en utilisant la taille et le niveau de gris de l'organe. Néanmoins ce dernier paramètre est variable selon l'âge des mouches (de La Rocque et al., 1996) et doit être considéré avec prudence.

Finalement, les différences des paramètres morphométriques permettent de classer $90 \%$ des mouches selon leur espèce et dans chaque espèce, plus de $90 \%$ des individus selon le sexe. Les sceptiques diront que cette classification est immédiate de visu, d'autres critères morphologiques étant évidents. C'est en effet le cas dans les sous-genres Nemorbina (groupe Palpalis) et Glossina (groupe Morsitans) mais au sein du sousgenre Austenina (groupe Fusca) la diagnose d'espèce exige l'examen des organes génitaux, technique complexe et fastidieuse (Itard, 1986). Une méthode morphométrique serait alors d'un intérêt certain, et les résultats présentés ici doivent être considérés comme une première étape pour le développement d'un outil rapide de diagnose, à tester sur ces espèces. Enfin, au sein même d'une espèce, de récents travaux ont permis de montrer le potentiel de cet outil pour les études de génétiques de population (Solano et al., 1999).

\section{LA TAILLE DES GLOSSINES ET LE MILIEU DE VIE}

Au sein d'un taxon donné, les facteurs environnementaux peuvent affecter la taille des glossines. Les effets de la température notamment ont été démontrés chez différents insectes (Brown, 1979; Burnik et al., 1988). Chez les tsé-tsé, les températures élevées conduisent à des populations de plus petite taille (Challier, 1973; Glasgow \& Bursell, 1961; Sané et al., 2000), comme nous l'observons également ici en saison chaude. Les conditions abiotiques auraient essentiellement un impact morphologique lors du stade nymphal, les températures élevées entraînant une augmentation de la durée pupale et une souffrance physiologique de la nymphe, obligeant l'insecte à puiser dans ses réserves lipidiques pour résister à la déshydratation (Bursell, 1960; Glasgow, 1970; Laveissière et al., 1984). La taille d'un individu est également liée à la longévité de sa mère et à son état nutritionnel. On sait que les vieilles femelles produisent une progéniture plus petite (De Deken et al., 1997; McIntyre \& Gooding, 1998). Les conditions écologiques rencontrées par la génération parentale déterminent les caractéristiques des populations suivantes, à travers l'abondance des réserves vitellines fournies à la larve. Par exemple, Dejardin et Maillot (1964) ont observé une corrélation positive entre la longueur du corps des tsé-tsé et 
l'humidité observée deux mois auparavant, période de la ponte. La taille des populations est ainsi un indicateur de l'état physiologique de la population précédente (Bursell, 1966; Buxton, 1955; Mulligan, 1970). Des variations morphométriques peuvent également être observées entre les populations issues de zones différentes, et soumises à des conditions climatiques graduelles. A une vaste échelle le long d'un transect de 700 kilomètres allant de Côte d'Ivoire jusqu'au Burkina Faso, Rogers et Randoph (1991) ont étudié des relations entre la taille de Glossina morsitans submorsitans (Newstead 1910) et les paramètres environnementaux appréhendés par télédétection. La taille des mouches a alors pu être corrélée au NDVI (Normalized Difference Vegetation Index) qui révèle l'activité photosynthétique de la zone étudiée. Les populations de moindre taille ont été collectées dans les zones les plus arides et ayant une couverture végétale faible. Notre étude a été menée à l'échelle de quelques dizaine de kilomètre, dans des conditions microclimatiques relativement homogènes. Lors de l'évaluation de l'effet de l'habitat, les deux populations étaient séparées d'une quinzaine de kilomètres seulement. Pourtant les glossines provenant de biotopes plus humides et ombragés (galeries forestières larges et fermées) étaient plus grosses que celles issues de cordons plus secs. En référence à ce qui a été dit plus haut, la taille est ainsi un indicateur de la qualité du biotope et de l'état de souffrance physiologique des populations locales, informations qui pourraient être utilisées pour adapter la pression de lutte antivectorielle. Certains auteurs ayant rapporté des différences dans la capacité de vol et dans la longévité des insectes selon leur taille (Dransfield et al., 1989; Dransfiels \& Brightwell, 1989; Rogers \& Randolph, 1991), ces relations pourraient également avoir un intérêt pour l'étude de la capacité vectorielle des populations locales et la description des sites de transmission.

\section{REMERCIEMENTS}

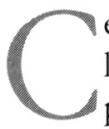

ette étude s'inscrit dans le cadre d'un projet sur l'épidémiologie des trypanosomoses, soutenu par le CIRAD-EMVT (Action Thématique programmée "Santé-Environnement") et le CNRS (Programme Interdisciplinaire "Environnement, Vies et Sociétés").

\section{RÉFÉRENCES}

Borne F., Petiteau L., Geoffroy B., De la Rocque S. \& CuiSANCE D. Fly Picture Measurement, un nouvel outil informatique pour l'étude des glossines. Revue d'Elevage et de Médecine Vétérinaire des pays Tropicaux, 1999, 52, Vol. 1, 19-21.
BROwn K.R. Comparative wing morphometrics of some calyprate diptera. Journal of the Australian entomological Society, 1979, 18, 289-303.

BRUCKNER D. The influence of genetic variability on wing symmetry in honeybees (Apis mellifera). Evolution, 1976, 30, 100-108.

Burnik M., Brncic D. \& M.A. Geographical and sexual variation of wing size in the monophagous flower-breeding species Drosophila flavopilosa. Zeitschrift für Zoologische Systematik und Evolutionforschung, 1988, 26, 211-216.

Bursell E. The measurement of size in tsetse flies (Glossina) Bulletin of Entomological Research, 1960, 51, 33-37.

Bursell E. The nutritional state of tsetse flies from different vegetation type in Rhodesia. Bulletin of Entomological Research, 1966, 57, 171-180.

Buxton P.A. The natural history of tsetse flies. An account of the biology of the genus Glossina (Diptera). 1955. London School of Hygiene and Tropical Medecine, Memoir $\mathrm{N}^{\circ}$ 10. Lewis H.K. \& Co Ltd, London, 816 p.

Chaldier A. Écologie de Glossina palpalis gambiensis Vanderplanck 1949 en savane d'Afrique Occidentale. 1973. Mémoire de l'ORSTOM n 64, Montpellier, 274 p.

Chaldier A. \& Laveissière C. Un nouveau piège pour la capture des glossines (Glossina: Diptera-Muscidae), description et essais sur le terrain. Cahiers de l'ORSTOM, Série Entomologie médicale et Parasitologie, 1973, 11, 251-262.

Daly H.V. Insect morphometrics. Annual Review of Entomology, 1985, 30, 415-438.

De Deken P., Van Den Bossche P., Sangare M., Gnanvi C., Missanda J.H. \& Van HeEs J. Effect of the life-span of female Glossina palpalis gambiensis on the weight and size of its progeny. Medical and Veterinary Entomology, 1997, 11, 95-101.

Dejardin, J. \& Maillot L. Biométrie de la glossine : Étude statistique des mensurations de l'aile dans diverses communautés (Glossina fuscipes quansensis, PIRES). Revue d'Elevage et de Médecine Vétérinaire des pays Tropicaux, 1964, 17, 97-102.

Dransfield R.D., Brightwell R., Killu J., Chaudhury M.F. \& ADABIE D.A. Size and mortality rates of Glossina pallipides in the semi-arid zone of southwestern Kenya. Medical and Veterinary Entomology, 1989, 3, 83-95.

Dransfield R.D. \& Brightwell R. Problems of field testing theoritical models; a case study. Annales de Société la Belge de Médecine Tropicale, 1989, 69 (suppl. 1), 147-154.

Dujardin J.P., Bermudez H. \& Schofield C.J. The use of morphometrics in entomological surveillance of sylvatic foci of Triatoma infestans in Bolivia. Acta Tropica, 1997, 66, 145-153.

GlasGow J.P. Selection for size in tsetse flies. Journal of Animal Ecology, 1961, 30, 87-94.

Glasgow J.P. The Glossina community, In: The African trypanosomiasis, Ed. Mulligan H.W., Allen \& Unwin, London, 1970, 348-381.

Glasgow J.P. \& Bursell E. Seasonal variations in the fat content and size of Glossina swynnertoni Austen. Bulletin of Entomological Research, 1961, 51, 705-718. 
Hoteluing H. Analysis of a complex of statistical variables into principal components. Journal of Educational Psychology, 1933, 417-441.

ITARD J. Les glossines ou mouches tsé-tsé. Études et Synthèses de l'IEMVT $\mathrm{n}^{\circ}$ 15, Maisons Alfort, 1986, $155 \mathrm{p}$.

LA RocQue S. (DE). Identification des facteurs discriminants de la présence de glossines dans une zone agro-pastorale du Burkina Faso. Intérêt pour l'évaluation du risque trypanosomien. Thèse Doct. Sci., Univ. Montpellier II, 1997, $162 \mathrm{p}$.

La Rocque S. (De), Geoffroy B. \& Cuisance D. Nouvelle approche pour l'estimation de l'âge des glossines par analyse d'image de l'aile. Revue d'Elevage et de Médecine Vétérinaire des pays Tropicaux, 1996, 49, 46-48.

Laveissière C., Kienou J.P. \& Traoré T. Écologie de Glossina tachinoides Westwood, 1850, en savane humide d'Afrique de l'Ouest. X. Durée du stade pupal. Importance de ce paramètre dans la dynamique des populations. Cabiers de l'ORSTOM, Série Entomologie médicale et Parasitologie, 1984, 22, 219-230.

Lebbe J., Torrez M.E., Vignes R. \& Dedet J.P. Wing morphology and species characterisation of sandflies. Parasitologia, 1991, 33, 365-372.

Mardia K.V., Kent J.T. \& BibBy J.M. Multivariate analysis. Academic Press, London, 519 p

MCINTYRE G.S. \& GOODING R.H. Effect of maternal age on offspring quality of tsetse (Diptera : Glossinidae). Journal of Medical Entomology, 1998, 35, 210-215.

Mulligan H.W. The African Trypanosomes. 1970. Allen G. \& Unwin LTD, London, $950 \mathrm{p}$.

Passerat de Silans L.N.M. Étude de la morphométrie alaire intra-spécifique et entre populations chez Phlebotomus perniciosus Newstead 1911 (Diptera: Psychodidae) par un système de mesures semi-automatiques. Rapport DEA, Univ. Montpellier I, 1994, 35 p.

Rogers D.J. \& Randolph S.E. Mortality rate and population density of tsetse flies correlated with satellite imagery. Nature, 1991, 351, 739-741.

Sané B., Solano P., Garcia A., Fournet, F. \& Laveissière C. Variations intraspecifiques de la taille des ailes et du thorax chez Glossina palpalis gambiensis (Robineau-Desvoidy, 1830) en zone forestière de Côte d'Ivoire. Revue d'Elevage et de Médecine Vétérinaire des Pays tropicaux, 2000, 53, 245-248.

Solano P., de la Rogue S., Cuisance D., Geoffroy B., de Meeüs T., Cuny G. \& Duvallet G. Intraspecific variability in natural populations of Glossina palpalis gambiensis from West Africa, revealed by genetic and morphometric analyses. Medical and veterinary Entomology, 1999, 13, 401-407.

Wall R. Ovarian ageing of tsetse flies (Diptera: Glossinidae) - Interspecific differences. Bulletin of Entomological Research, 1990, 80, 85-89.

Weeks P.J.D., Gauld I.D., Gaston K.J. \& O’Neill M.A. Automating the identification of insects: a new solution to an old problem. Bulletin of Entomological Research, 1997, 87, 203-211.

Reçu le 5 septembre 2001

Accepté le 10 mai 2002 